\title{
Fractal Roughness Effects on Nanoscale Grinding
}

\author{
Michail Papanikolaou, Konstantinos Salonitis \\ Manufacturing Theme, Cranfield University, Cranfield MK43 OAL, United Kingdom
}

\begin{abstract}
Three-dimensional Molecular Dynamics simulations have been performed to investigate the effects of fractal roughness on nanoscale grinding. The first part of this investigation focuses on the effects of the workpiece rough top surface on the grinding process characteristics, with special emphasis placed on the friction coefficient, the grinding forces and the subsurface temperature. The second part focuses on the alternation of the aforementioned parameters due to the rough abrasive contact surface. Rough surface profiles have been generated using the multivariate Weierstrass-Mandelbrot function while the abrasive has been modelled as a trapezoid. The irregular surface topography has been controlled by tuning the value of the fractal dimension $D_{s}$. The aforementioned experiments have been repeated for various values of the cutting depth. Our results indicate that the grinding process parameters are mainly dependent on the cutting depth as well as the abrasive lower surface profile, which also defines the interface contact area between the workpiece and the abrasive, rather than the topography of the workpiece top surface.
\end{abstract}

Keywords: Roughness, Fractal Geometry, Molecular Dynamics, Grinding

\section{Introduction}

The continuous growth of the Microelectromechanical Systems (MEMS) production as well as their application range has been the spark for the development of nanomachining technologies. The core goal of those technologies is the fabrication of high quality and ultra-precision miniaturised devices. During the last decades in-depth research has been conducted to allow for the enhancement of the current material removal processes. However, it has been pointed out that experimental investigations of nanomachining processes are limited by the accuracy and resolution of the actuation and sensor systems mounted on the machine tools [1]. In order to overcome these limitations a wide variety of computational and simulation models has been developed to shed light in the mechanics of abrasive processes [2]. Two of the most important methods employed for the investigation of the 
material removal processes' mechanics and thermodynamics are Kinematic Models and Finite Element Analysis (FEA). Since the 1980's the increasing computing power allowed for the development of Kinematic-Geometrical Models which enhanced our understanding of the process mechanics and contributed towards the optimisation of the process [3]. Although Kinematic Models can be used for the estimation of the physical process characteristics such as the forces and temperature, FEA is highly favourable for this purpose. FEA has been widely implemented for investigating the grinding process characteristics both in microscale [4] and in macroscale [5]. Despite their wide applicability and their successful implementation in a number of cases the aforementioned methods suffer in terms of accuracy when approaching micro- and nanoscale. This is because in such small scales the surface-area-tovolume ratio obtains larger values and surface phenomena become increasingly dominant [6].

This issue can be addressed with the help of the Molecular Dynamics (MD) simulation technique which uses atomistic models and can provide intellectual transparency and a deep insight into the phenomena taking place in nanoscale compared to methods such as Kinematic Models and FEA. As expected though, the added resolution of MD is offset by additional CPU demand. MD simulations have been extensively employed for simulating nanocutting processes as they can accurately and consistently describe the mechanical and thermal properties of the materials under investigation and the physics of the processes. The first MD simulations on material removal have been conducted by Landman et al. [7]. Since then, many researchers have used MD for developing qualitative and quantitative understanding of abrasive processes over a wide range of material combinations and process parameters. One of the examined topics using MD has been the investigation of the effect of the tool geometry on the grinding mechanics. Komanduri et al. [8] performed 2-dimensional MD simulations and modelled the abrasive as an edge with a radius $r$ and a clearance angle. They proposed that the cutting force increases while the specific energy decreases with increasing depth of cut $d$, independently of the $d / r$ ratio for a specific range of $d / r$ ratios $(0.1-0.3)$. The effect of the tool geometry on the chip formation and cutting forces has been investigated by Pei et al. [9]. They suggested that the chip thickness becomes smaller while the cutting force obtains higher values as the tool rake angle increases from $-45^{\circ}$ to $45^{\circ}$. Moreover, the authors compared the results obtained by using the Morse [10] and Embedded Atom Method (EAM) [11] potentials respectively. They concluded that the Morse potential leads to overestimation of the cutting forces and therefore suggested that the EAM potential should be employed for nanocutting MD simulations. The Face Centred Cubic (FCC) crystal orientation effects on cutting forces, dislocation movement and material deformation have been studied

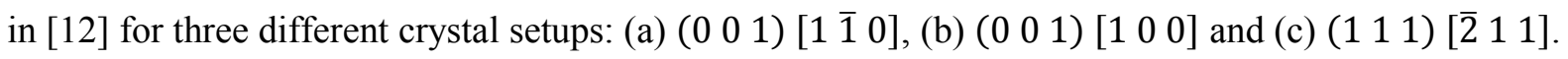
It was found that, for the aforementioned crystal orientations, dislocations propagate at $0^{\circ}, 45^{\circ}$ and $60^{\circ}$ respectively. Moreover, the lowest cutting forces were observed in case (a), while the highest cutting forces in case (c). The investigation of subsurface damage by means of MD simulation in grinding has recently come into the spotlight. $\mathrm{Li}$ et al. [13] simulated the grinding process of a monocrystalline 
silicon workpiece and identified that, as the abrasive advances, there is a phase transformation of the silicon structure from stable diamond cubic (alpha-silicon) to body centred tetragonal (beta-silicon). Moreover, their results showed that there is a dependence between the subsurface damage (SSD) and the grinding velocity, with higher cutting velocity inducing larger chip volume and consequently lower SSD thickness.

Despite of the numerous MD studies on grinding processes, the effects of surface roughness on the process mechanics is a topic that has not been thoroughly investigated. Li et al. [14] performed MD simulations to shed light on the effects of a copper workpiece surface roughness on the nanocutting process characteristics. In order to describe surface roughness they used orthogonal asperities. Their results indicated that the cutting forces as well as the friction coefficient are dependent on the texture density $\rho=\frac{R_{x}}{R_{0}}$, where $R_{x}$ is the width of a single asperity and $R_{0}$ the distance separating two subsequent asperities. Their results indicated that both cutting forces and friction coefficient present an increasing trend with increasing texture density. Moreover, the effect of a nanostructured abrasive with rectangular grooves on the induced stresses, phase transformation, cutting temperature, friction coefficient and cutting forces has been studied by Dai et al. [15]. Periodical protrusions have been broadly used in MD simulations for representing rough surface topographies [16,17]. However, scientific reports have demonstrated that many surface types, including diamond, follow a self-affine pattern in reality, i.e. they present similar features over a range of scales $[18,19]$. Moreover, it has been reported that at the nanoscale the surface of most materials are fractals with a fractal dimension within the range $2 \leq D_{s} \leq$ 3 [20]. Therefore, more recent studies have employed fractal theory, instead of Euclidean geometry, to accurately represent irregular surfaces both in 2 [21] and 3 dimensions [22,23]. However, up to now, there have been no MD investigations using fractal theory for modelling abrasive processes.

In this study, we have employed the Molecular Dynamics simulation method to investigate the effects of both workpiece and abrasive surface roughness in nanocutting. We have used the multivariate Weierstrass-Mandelbrot function [24] to generate stochastically rough surface profiles with varying complexity by controlling the value of the fractal dimension $D_{s}$. Our results indicate that surfaces with more irregular interface contribute towards the increase of the cutting forces, the friction coefficient as well as the temperature in the vicinity of the abrasive. Moreover, the workpiece top surface morphology does not affect significantly the grinding process characteristics. The cutting forces along with the subsurface temperature and the friction coefficient increase with the cutting depth. Based on our analysis, it is strongly recommended that abrasive materials with low fractal dimension values are being used in order to reduce the cutting forces and subsequently the subsurface temperature and damage. Another solution could be controlling the abrasive surface roughness through recently developed nanopatterning techniques [25]. 


\section{Simulation Method}

Our MD model consists of a trapezoidal diamond abrasive and a copper workpiece. This combination of materials has been extensively used across the literature and the interaction potentials have been well established $[9,14]$. The dimensions of the abrasive and the workpiece are listed in Table 1Error! Reference source not found.. The workpiece and abrasive atoms are placed on the sites of a Face Centred Cubic (FCC) lattice (lattice constant equal to $3.597 \AA$ ) and a diamond lattice (lattice constant equal to $3.57 \AA$ ) respectively. There are 3 atom types contained in the simulation domain: (a) Newtonian atoms which move according to the second Newton's law, (b) thermostat atoms on which Langevin thermostats, each one composed of 5 atom layers [26], are being applied to for maintaining a constant temperature equal to $300 \mathrm{~K}$ across the simulation domain and (c) boundary atoms, as illustrated in Figure 1. The workpiece boundary atoms are fixed to their initial positions while the abrasive boundary atoms start moving with a constant velocity equal to $100 \mathrm{~m} / \mathrm{s}$, which falls into the high speed grinding process specifications [27], after the equilibration phase has been completed. Grinding is

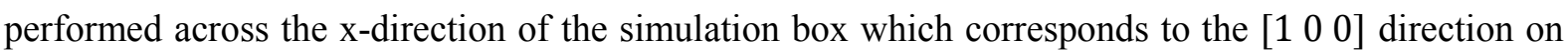
the $\left(\begin{array}{lll}0 & 0 & 1\end{array}\right)$ plane of the workpiece.
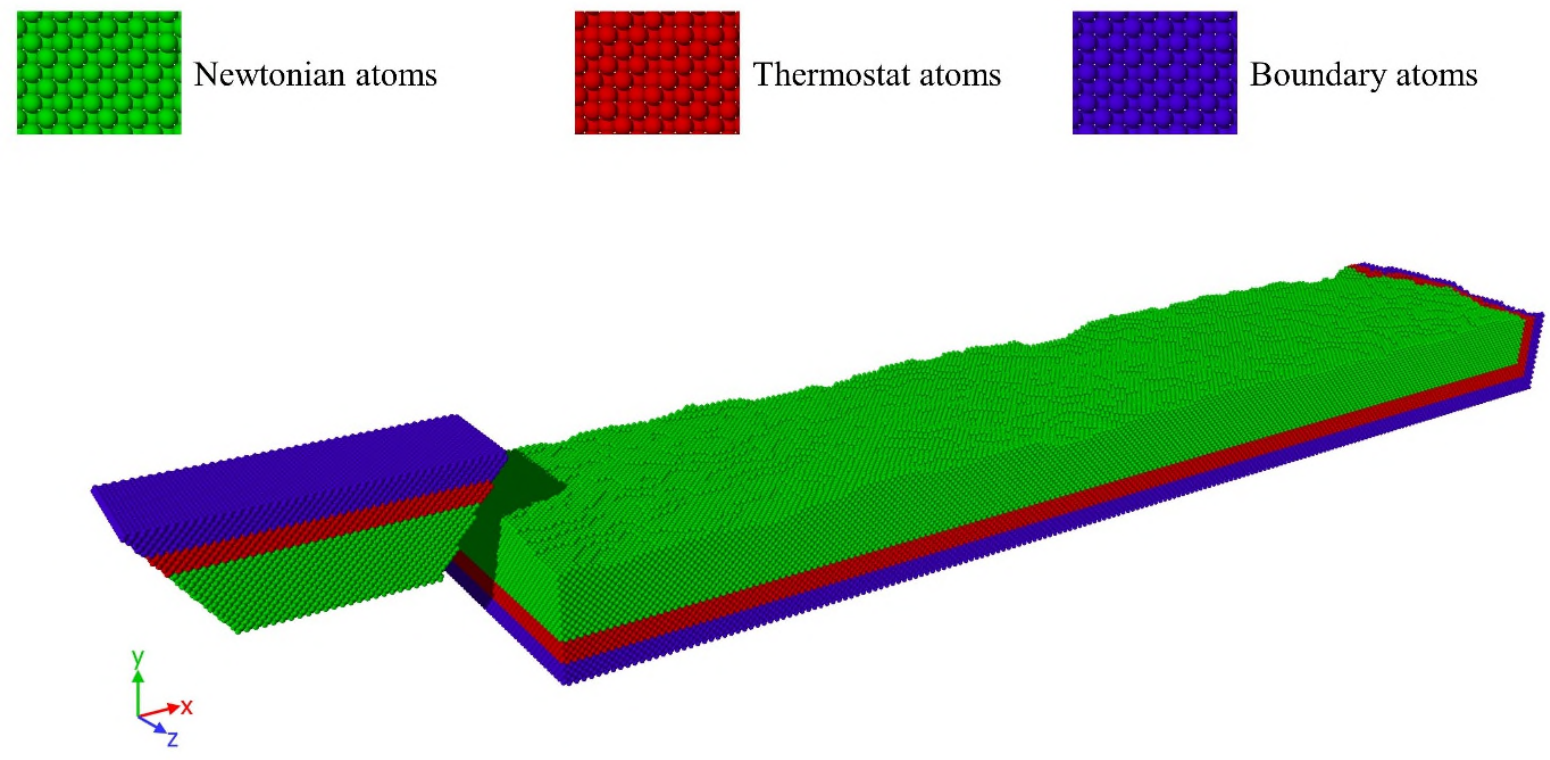

Figure 1: Simulation domain

There are 3 different interaction types across the simulation domain between the: (a) abrasive diamond atoms $(\mathrm{C}-\mathrm{C}),(\mathrm{b})$ workpiece copper atoms $(\mathrm{Cu}-\mathrm{Cu})$ and $(\mathrm{c})$ abrasive and workpiece atoms $(\mathrm{C}$ $\mathrm{Cu}$ ) which have been described by three different interaction potentials, similarly to [14]. The interactions between the abrasive diamond atoms $(\mathrm{C}-\mathrm{C})$ have been described using the Tersoff potential which can provide an accurate description of the structural properties of diamond [28]: 


$$
\begin{gathered}
E=\frac{1}{2} \sum_{i} \sum_{i \neq j} V_{i j} \\
V_{i j}=f_{c}\left(r_{i j}\right)\left[f_{R}\left(r_{i j}\right)+b_{i j} f_{A}\left(r_{i j}\right)\right]
\end{gathered}
$$

where $E$ is the total energy of the system of atoms, $V_{i j}$ the bond energy between atoms $i$ and $j, f_{c}$ the cutoff function, $f_{A}$ an attractive pair potential, $f_{R}$ a repulsive pair potential and $b_{i j}$ a monotonically decreasing function of the coordination of atoms $i$ and $j$.

Table 1: Characteristic dimensions

\begin{tabular}{|l|c|l|c|}
\hline \multicolumn{2}{|c|}{ Workpiece } & \multicolumn{2}{c|}{ Abrasive } \\
\hline Length (x-dimension) & $790 \AA$ & Upper Edge (x-dimension) & $155 \AA$ \\
\hline Height (y-dimension) & $54 \AA$ & Lower Edge (x-dimension) & $86 \AA$ \\
\hline Width (z-dimension) & $124 \AA$ & Height (y-dimension) & $50 \AA$ \\
\hline & Width (z-dimension) & $40 \AA$ \\
\hline
\end{tabular}

The interactions between the copper atoms of the workpiece have been described using the EAM potential which is one of the most common, simple and accurate models of atomic bonding in metals [11]:

$$
v_{i}^{E A M}=\sum_{j=1}^{N_{i}} v_{i j}+f\left(\rho_{i}\right)
$$

where $v_{i j}$ is the interaction energy between atoms $i$ and $j, \rho_{i}$ is the local electron density and $f$ an embedding function.

Finally, the interaction between the copper and diamond atoms of the workpiece and the abrasive respectively, has been modelled with the Morse potential which has been effectively used in the past for describing the interaction between copper and diamond atoms [29]:

$$
E=D_{0}\left[e^{-2 a\left(r-r_{0}\right)}-2 e^{-2 a\left(r-r_{0}\right)}\right]
$$

where $E$ is the pair interaction energy $D_{0}=0.087 \mathrm{eV}$ the cohesion energy, $a=5.14$ elastic modulus and $r_{0}=2.05 \AA$ the equilibrium distance [30].

The 3-dimensional rough surface profiles have been generated using the multivariate Weierstrass-Mandelbrot function [31] which has been widely used for modelling the elastic contact between 3-dimensional fractal surfaces [18]: 


$$
\begin{gathered}
y(x, z)=C \sum_{m=1}^{M} \sum_{n=0}^{n_{\max }} \gamma^{\left(D_{s}-3\right)}\left\{\cos \Phi_{m, n}-\cos \left[\frac{2 \pi \gamma^{n} \sqrt{\left(x^{2}+z^{2}\right)}}{L_{M A X}} \cdot \cos \left(\tan ^{-1}\left(\frac{z}{x}\right)-\frac{\pi m}{M}\right)+\Phi_{m, n}\right]\right\} \\
C=L_{M A X}\left(\frac{G}{L_{M A X}}\right)^{D_{s}-2}\left(\frac{\ln \gamma}{M}\right)^{1 / 2}
\end{gathered}
$$

This function embeds $M$ surfaces; each one is a superposition of $n_{\max }$ different frequencies. Each frequency on each surface, indexed by the integers $n$ and $m$ respectively, is offset by a random phase $\Phi_{m, n}$. The value of $\gamma$ defines the frequency density and $L_{M A X}$ is the size of the sample. The most typical values of $\gamma$ are 1.5 and 5 according to the literature. In this study, we have used $\gamma=1.5$ which generates a surface with moderate separation of roughness scales [24]. $L_{M I N}$ is the cutoff length. The parameter $D_{s}$, which is under investigation in this study, is the fractal dimension; an indication of the fractal's ability to fill up space. For a 3-dimensional fractal the values of the fractal dimension are in the range of $2<D_{s}<3 . G$ is a height scaling parameter, independent of the roughness frequency. Finally, $C$ is a scaling factor determining the average amplitude of the cosine waves. In theory, a perfect fractal corresponds to an infinite number of frequencies, i.e. $n_{\max } \rightarrow \infty$. However, an appropriate value can be also selected by using:

$$
n_{\text {max }}=\operatorname{int}\left[\frac{\log \left(L_{\max } / L_{\min }\right)}{\log \gamma}\right]
$$

The effects of the aforementioned parameters on the final surface topography are well illustrated in [31].

$D_{s}=3.0$
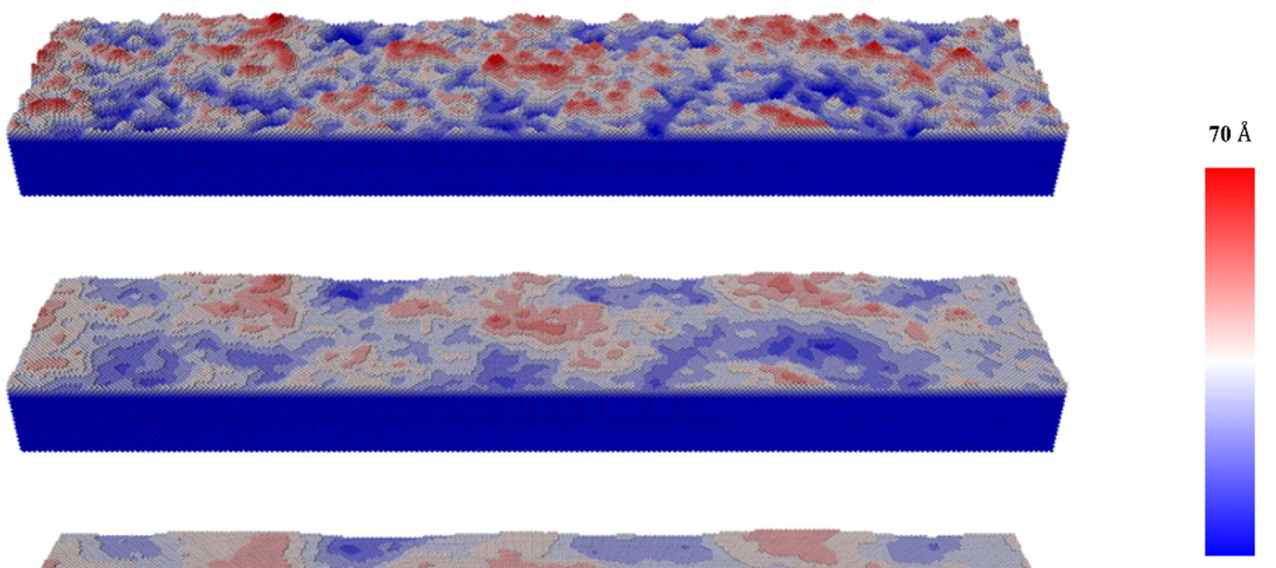

$D_{s}=2.0$
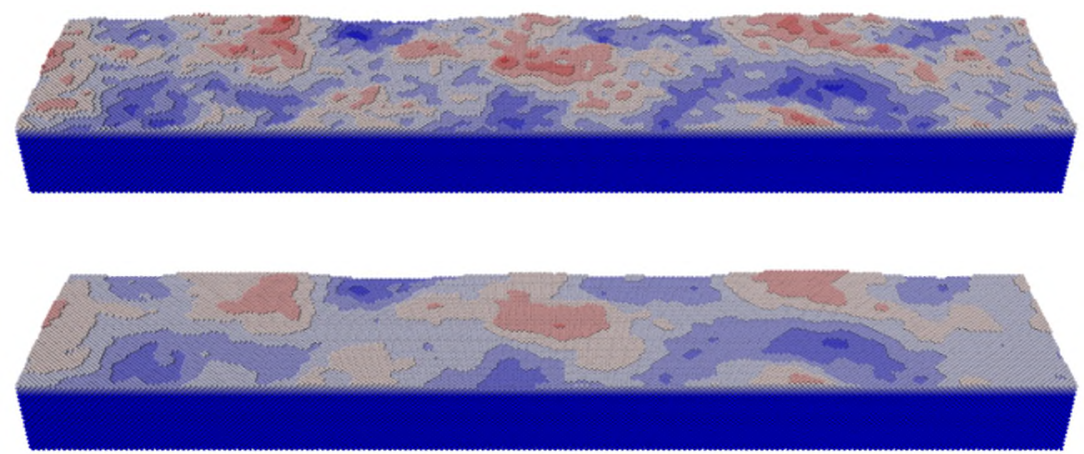

Figure 2: Effect of fractal dimension on the workpiece surface topography

In this study, we have been constantly adjusting the value of $G$ in order to maintain the average amplitude of the waves $C$ constant, as the fractal dimension obtained different values. A set of surfaces 
generated using 3 different values of the fractal dimension for the workpiece and the abrasive is depicted in Figure 2. It is apparent that higher values of the fractal dimension $D_{s}$ correspond to increasingly irregular topographies.

Initially, the simulation domain is equilibrated for 60,000 timesteps using the NVE ensemble, until a constant temperature equal to $300 \mathrm{~K}$ and a constant pressure is achieved. The timestep has been set equal to $0.001 \mathrm{ps}$. After the equilibration phase has been completed, a constant velocity equal to 100 $\mathrm{m} / \mathrm{s}$ is imposed on the fixed boundary atoms of the abrasive along the $\mathrm{x}$-direction of the simulation box.

As stated in the Introduction paragraph, fractals should be used instead of Euclidean geometry to realistically describe natural surfaces. In this study, both the workpiece and abrasive roughness effects on the grinding process have been studied. For both of the aforementioned cases, three different values of the cutting depth, which has been measured from the roughness centreline of the workpiece or the abrasive (Figure 3), have been used. Moreover, the maximum roughness amplitude is always smaller than $d_{c} / 2$, so as to ensure that there is perfect contact between the two bodies.

(a) Rough workpiece surface

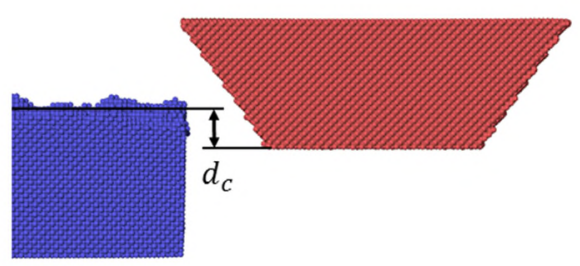

(b) Rough abrasive surface

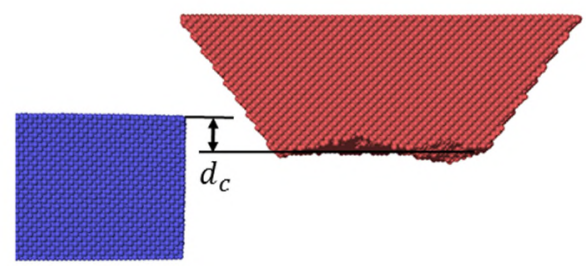

Figure 3: Depth of cut

\section{Results and Discussion}

The objectives of this investigation are the identification of the effects of the cutting depth and the fractal roughness, being tuned using the fractal dimension, on the following grinding process characteristics: (a) cutting forces, (b) friction coefficient and (c) subsurface temperature. For the purpose of the current analysis we have considered both the effects of the abrasive and the workpiece roughness. In the following paragraphs we will present the effects of the grinding surfaces' irregularities and the cutting depth on the process characteristics and try to shed light on the nanoscale phenomena inducing the observed behaviour.

\subsection{Surface roughness effects on the grinding process characteristics}

\subsubsection{Grinding forces}

The total grinding force consists of 3 components, namely rubbing, ploughing and cutting [32]. Figure $4 \mathrm{a}$ shows the evolution of the grinding forces during a grinding simulation. It is evident that both 
of the cutting forces $\left(F_{x}\right.$ and $\left.F_{\mathrm{y}}\right)$ gradually increase until they obtain a constant value. This occurs when the abrasive has totally penetrated into the workpiece and consequently the maximum contact area between the two rigid bodies has been achieved. It can be observed that, after the point in time when steady state has been reached, there are some oscillations in the grinding forces. This is consistent with previous MD [12] and FEM [4] simulations.
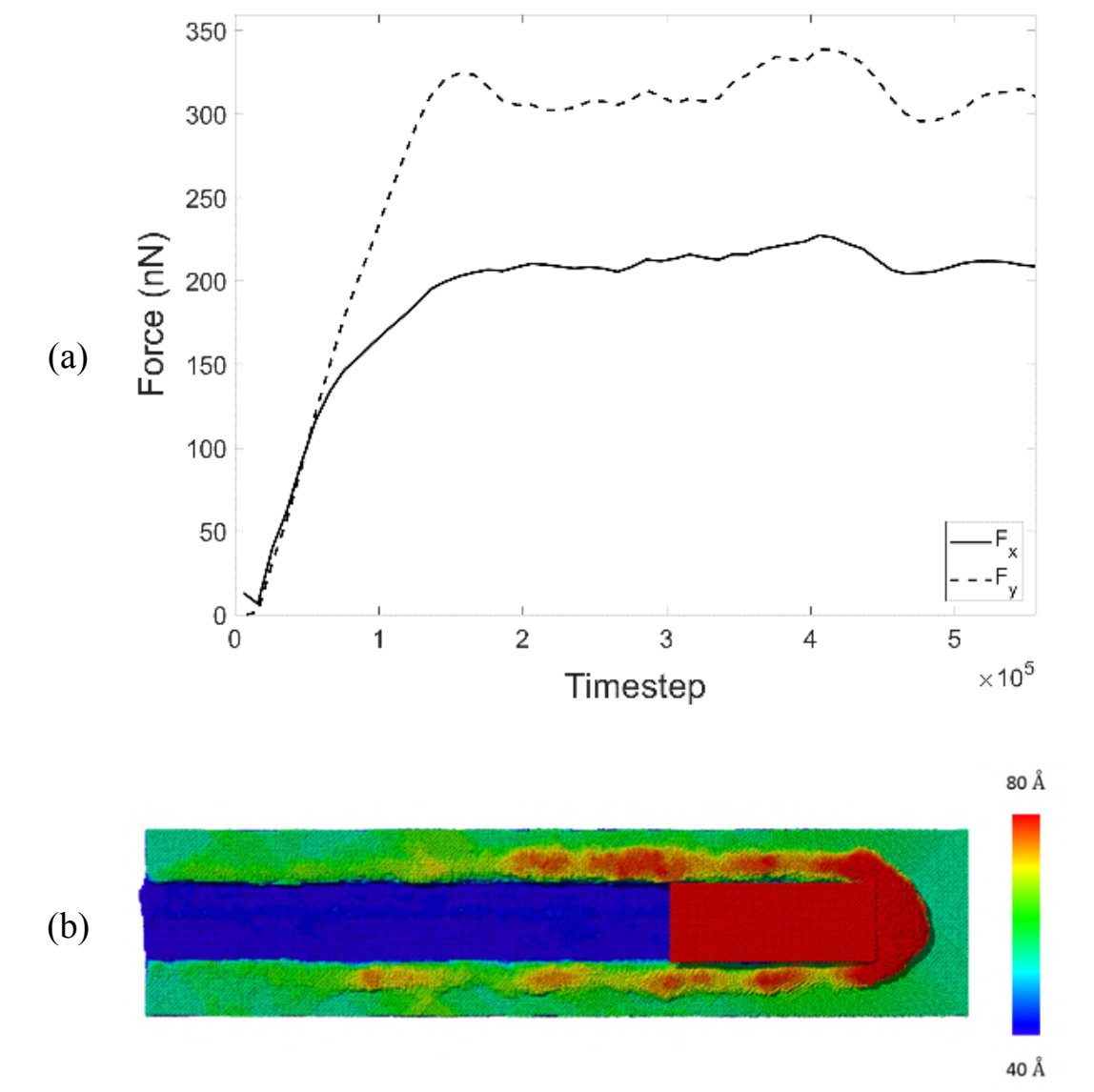

Figure 4: Evolution of (a) cutting forces and (b) workpiece surface topography during grinding $\left(D_{s, \text { workpiece }}=3.0\right)$

Figure 5 illustrates the effect of workpiece roughness on the grinding forces. It can be observed that both the tangential and normal forces follow the exact same pattern, with the only difference that $F_{y}>F_{x}$, and oscillate around constant values. It seems that their mean values are not dependent on the workpiece surface irregularity. The large fluctuations of the grinding forces are associated with the generation of dislocations according to Lin et al. [33]. Moreover, it is obvious that the normal forces undergo more intense fluctuations in terms of magnitude compared to the tangential forces. This is attributed to the higher magnitude of the normal forces compared to the magnitude of the tangential ones. Previous investigations have shown that for higher values of the cutting depth the magnitude of the tangential and normal forces and consequently the magnitude of their fluctuations are about equal [34]. 
(a)

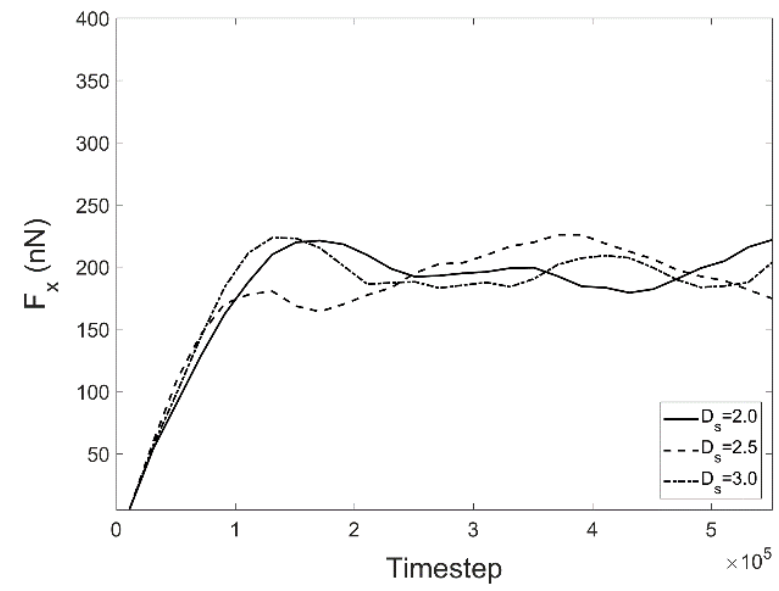

(b)

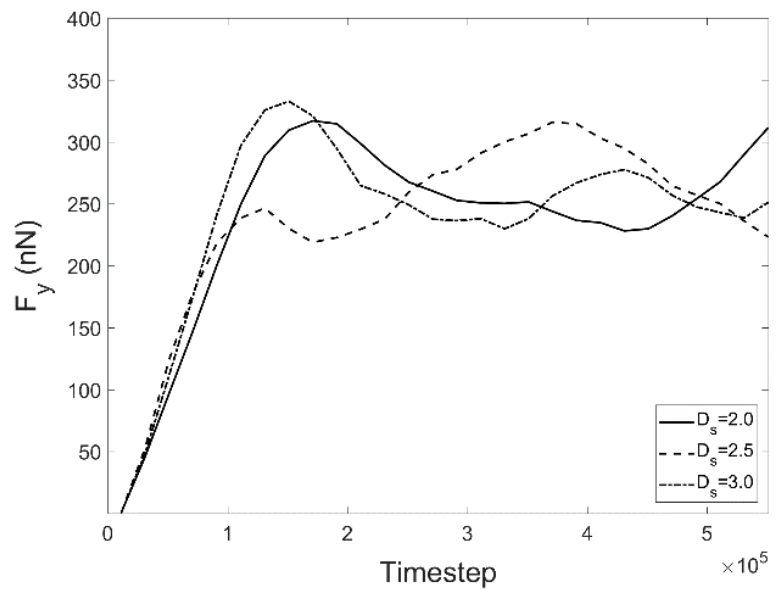

Figure 5: (a) Tangential and (b) normal forces vs time as a function of the fractal dimension of the workpiece surface

In order to study the dependency of the mean values of the cutting forces on the fractal dimension of the workpiece rough surface we performed 3 simulations for each value of the fractal dimension $D_{S}$ in favour of statistical accuracy, i.e. 9 simulations in total. For each value of the fractal dimension we selected a different random phase matrix $\Phi_{m, n}$ (Eq. 4). The mean grinding forces were estimated by averaging their values over the last 300,000 timesteps of each simulation. Figure 6 reveals that the cutting forces are approximately insensitive to the fractal dimension of the rough workpiece surface and they maintain a constant value. This is because the interface between the workpiece and the abrasive is smooth in spite of the rough workpiece top surface. The average forces computed in this study are generally larger compared to previous studies with the same material combination [12]. The differences observed are due to the different (a) contact area between the workpiece and the abrasive, (b) depth of cut, (c) cutting speed and (d) abrasive geometry.

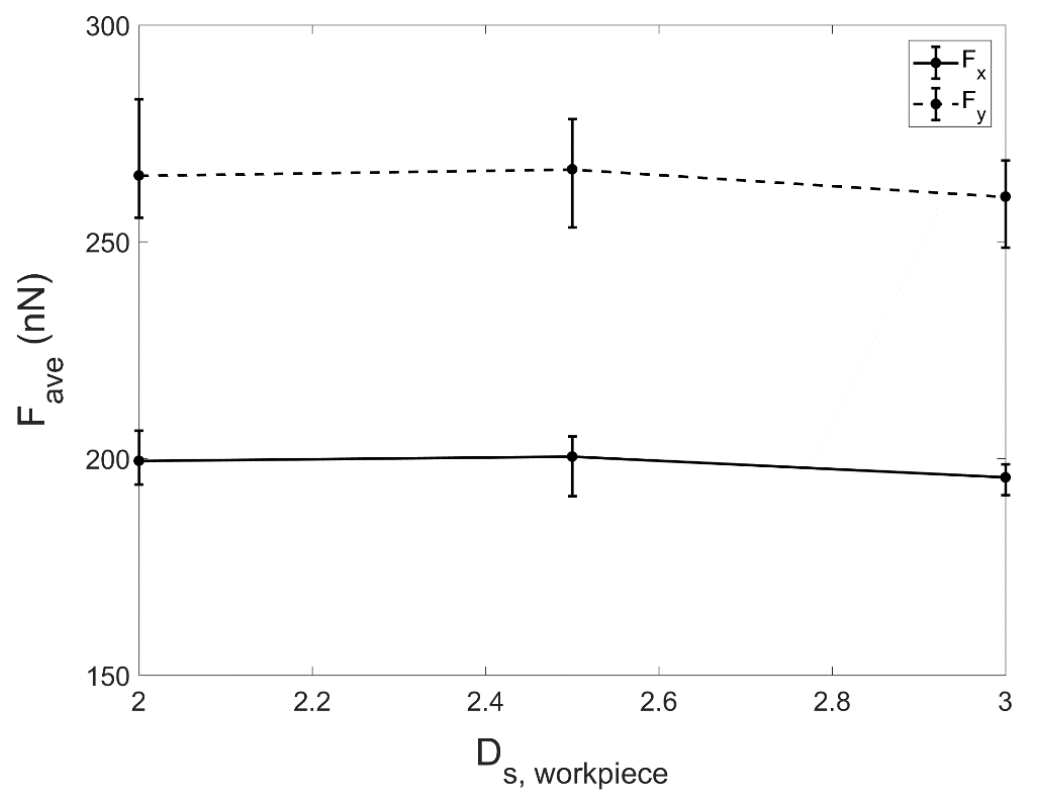

Figure 6: Mean tangential and normal forces vs fractal dimension of the workpiece surface 
However, the situation changes completely when roughness is introduced in the abrasive lower surface. Figure 7 illustrates the effects of the abrasive surface irregularities on the cutting forces. In Figure $7 \mathrm{a}$ we can see that there is a significant increase in the tangential force $F_{x}$ for higher values of $D_{s}$ while the normal force $F_{y}$ seems to be slightly increasing with the value of the fractal dimension (Figure 7b).

(a)

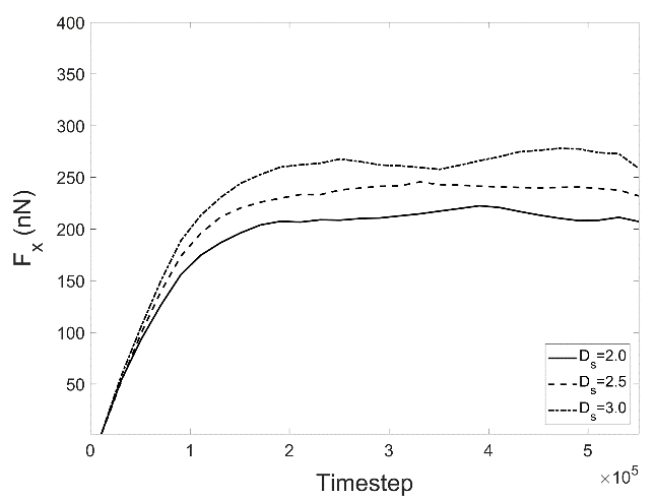

(b)

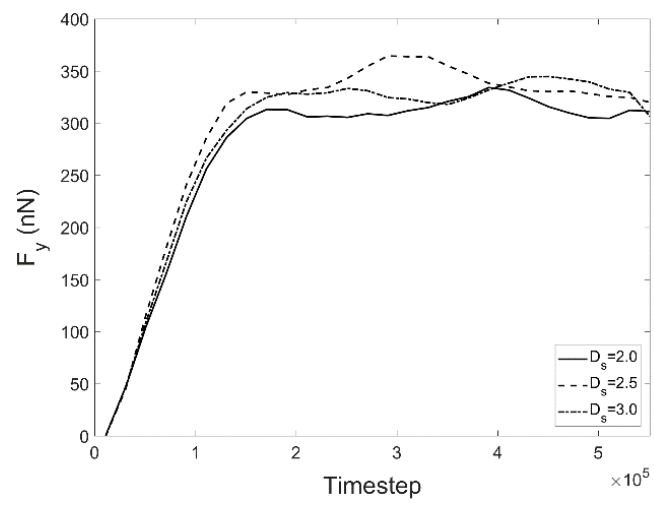

Figure 7: (a) Tangential and (b) normal forces vs time as a function of the fractal dimension of the abrasive surface

Similarly to the rough workpiece surface case, 9 simulations were performed in order to reduce the statistical error by using different values of the random phase $\Phi_{m, n}$ of the W-M function. Our results show that the tangential component of the mean grinding force is approximately linearly increasing with the value of the fractal dimension (Figure 8). The increase of the tangential forces with the fractal dimension is attributed to the stronger interlocking mechanism between the workpiece and abrasive surface for more irregular abrasive surface topographies. A similar trend is observed in the mean value of the normal force with the difference that the increase is smoother. This is because the number of asperities increases for higher values of the fractal dimension.

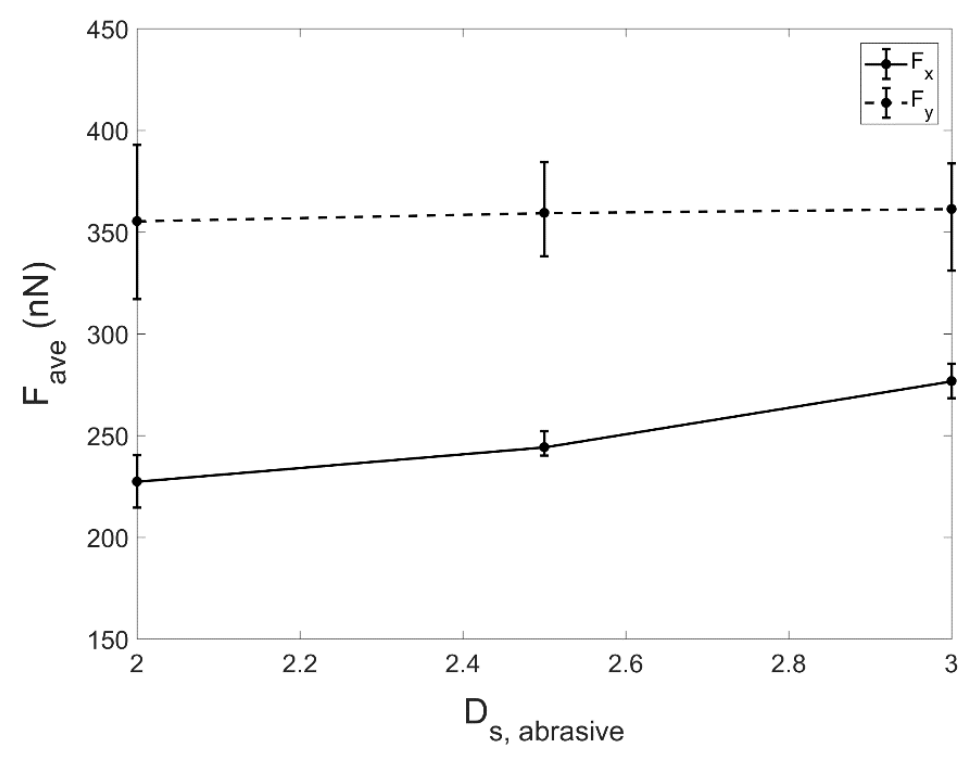

Figure 8: Mean tangential and normal forces vs fractal dimension of the abrasive surface 


\subsubsection{Friction Coefficient}

Similarly to the evolution of forces, the friction coefficient $\eta$ obtains a constant value after the abrasive has completely penetrated into the workpiece. However, as depicted in Figure 9, a different trend is evident in this case; the friction coefficient decreases gradually until it reaches a constant value. This trend is consistent with the FEA results of Mistakidis et al. [35]. They attributed this phenomenon to the large resistance encountered in the first steps provided by the locking of the asperities. It can be observed that when the interface area between the abrasive and the workpiece is small, during the initial timesteps, the friction coefficient can obtain values even greater than 1.0, which is rare in macroscale. However, as the interface area increases, $F_{y}$ increases correspondingly, leading to a reduction in the friction coefficient. At this point, it has to be noted that the average friction coefficient is dependent on the abrasive geometry and it is possible that its mean value can become higher than 1.0, especially when the interface area is small compared to the cutting depth [14].

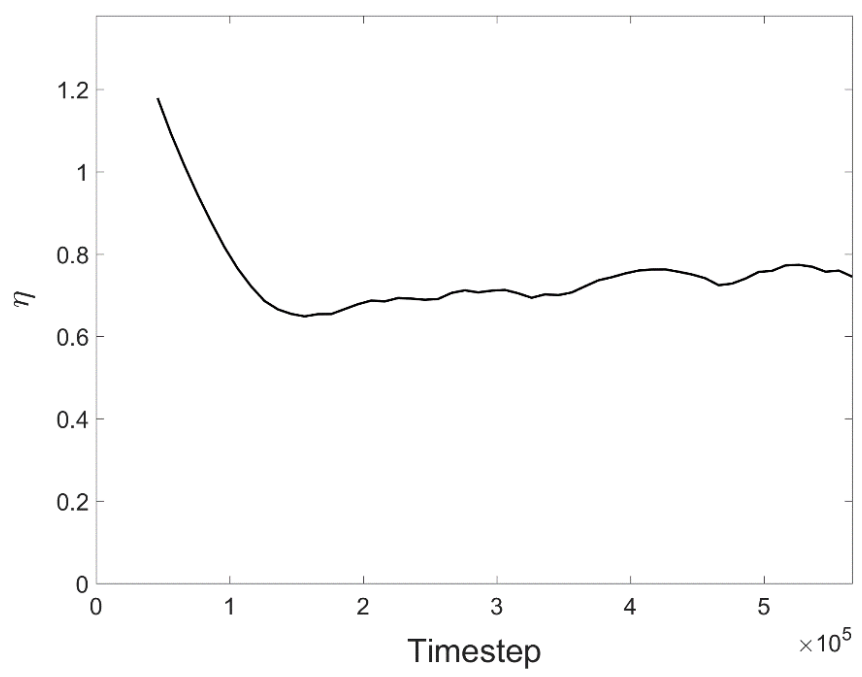

Figure 9: Evolution of the friction coefficient $\left(D_{\text {s,workpiece }}=3.0\right)$

Following the procedure described in the previous paragraph we evaluated the mean value of the friction coefficient for the rough workpiece and abrasive cases. The average friction coefficient was calculated as the average of the fraction of the tangential to the average normal force $\left(\eta=\left\langle\frac{F_{x}}{F_{y}}\right\rangle\right)$ over the last 300,000 timesteps of each simulation. As expected, the friction coefficient in the rough workpiece case was found to be insensitive to the workpiece surface irregularity (Figure 10). This behaviour is attributed to the smooth interface between the workpiece and the abrasive and is in agreement with the experimental work of Menezes et al. [36]. On the contrary, the friction coefficient depends on the irregularity of the abrasive surface and increases with its fractal dimension. For an almost perfect fractal $\left(D_{s}=3\right)$ the friction coefficient becomes about equal to the corresponding value $(\cong 0.75)$ in the rough workpiece case, where the interaction surface between the abrasive and the workpiece is macroscopically smooth. This is of particular interest if we consider that an atomically 
smooth surface actually consists of atoms placed on discrete lattice positions and corresponds to a fractal surface profile with a small value of the scaling constant $C$ and $D_{S}=3$ [37]. In the case of a completely smooth interface both the tangential and normal force obtain their minimum values as there is approximately no interlocking and the contact area is the lowest possible correspondingly. The increase of the friction coefficient with increasing fractal dimension of the interface is consistent with the results of the FEM model presented by Panagouli et al [38] and is attributed to the stronger interlocking mechanism between the two surfaces for higher values of the fractal dimension. More specifically, the increased number of asperities associated with higher values of the fractal dimension leads to higher contact area between the two solids.

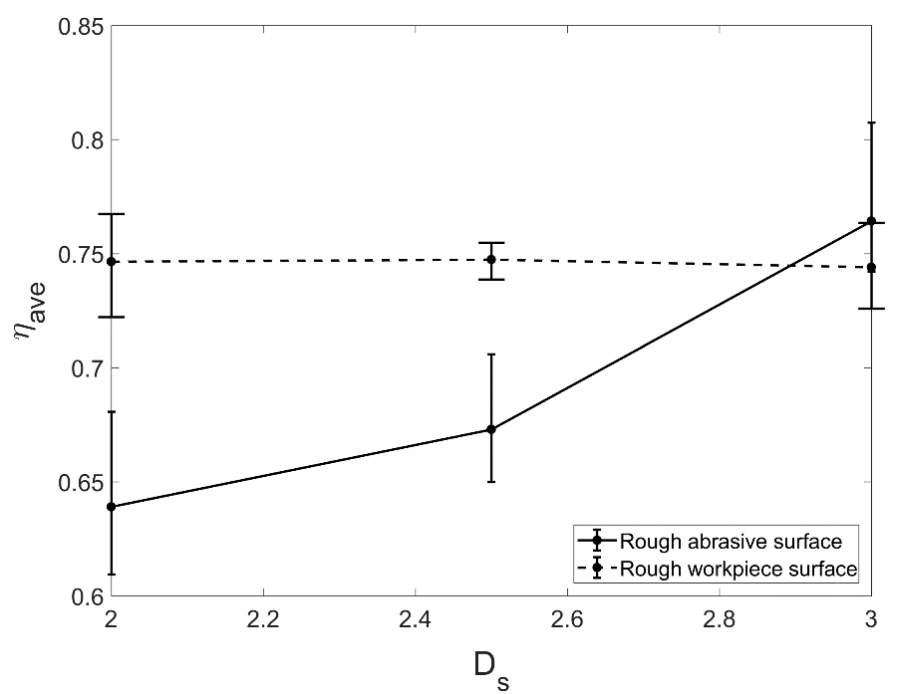

Figure 10: Mean friction coefficient for the rough workpiece and abrasive cases vs fractal dimension for the rough workpiece and abrasive cases

\subsubsection{Temperature}

In contrast to the evolution of the cutting forces and the friction coefficient, the simulation box temperature approaches asymptotically a constant temperature (Figure 11a \& b). This is because additional time is needed for the Langevin thermostats to subtract the induced heat from the simulation box. In contrast to the rough workpiece surface case (Figure 11a), we can observe that there is an increase in the simulation box temperature with the abrasive surface fractal dimension (Figure 11b). This result is in agreement with the observations of Dai et al. [15].

It is of particular interest to examine the workpiece temperature in the vicinity of the abrasive grain. In order to calculate the temperature in this area we defined a spherical region with a radius equal to $10 \AA$ and its centre positioned at the centre of mass of the abrasive. This region was set up as a moving region in order to follow the abrasive trajectory while the workpiece atoms lying within the region were updated every 1 timestep. The temperature of the workpiece atoms surrounded by this region was calculated according to: 


$$
T=\frac{K E}{\left(\frac{\operatorname{dim}}{2} N k_{B}\right)}
$$

where, $K E=\sum_{i=1}^{n} \frac{1}{2} m_{i} v_{i}{ }^{2}$, is the total kinetic energy of the group of atoms, $m_{i}$ the mass of the atom $i, v_{i}$ the z-component of the velocity, $k_{B}$ the Boltzmann constant and $N$ the total number of workpiece atoms contained by this region. To obtain an accurate measurement of the temperature we used only the z-component of the velocity $(\operatorname{dim}=1)$ as the other 2 components are associated with the kinetic energy induced by the movement of the abrasive (x-direction) and the chip formation (y-direction). For the estimation of the temperature at this region we performed time-averaging over a window of 2,000 timesteps. The temperature in this region reaches an equilibrium state much faster than the temperature in the entire simulation box because the atoms lying in this region are constantly being updated (Figure $11 \mathrm{c} \& \mathrm{~d}$ ). Moreover, the temperature in the vicinity of the abrasive is about $100 \mathrm{~K}$ higher compared to the temperature of the simulation box. This is because the moving interface between the two solids acts like a heat source; consequently, the atoms lying closer to this heat source have higher temperature compared to the rest of the atoms. The trends of these graphs are consistent with the ones followed by Figure 11a \& b.

(a)

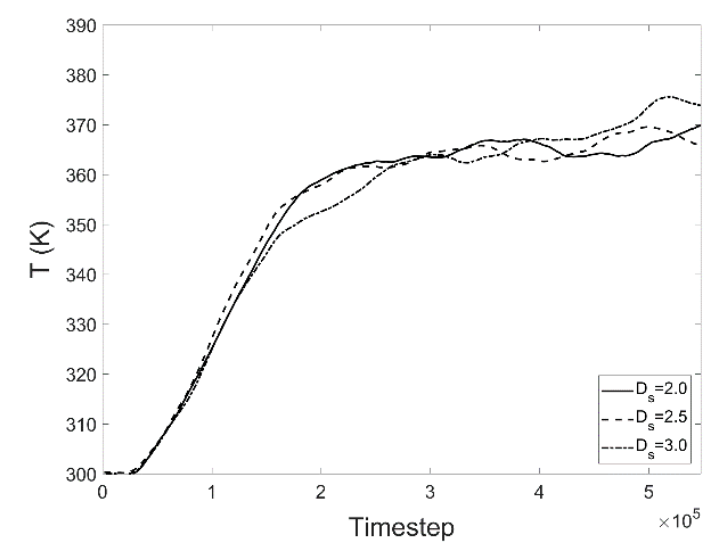

(c)

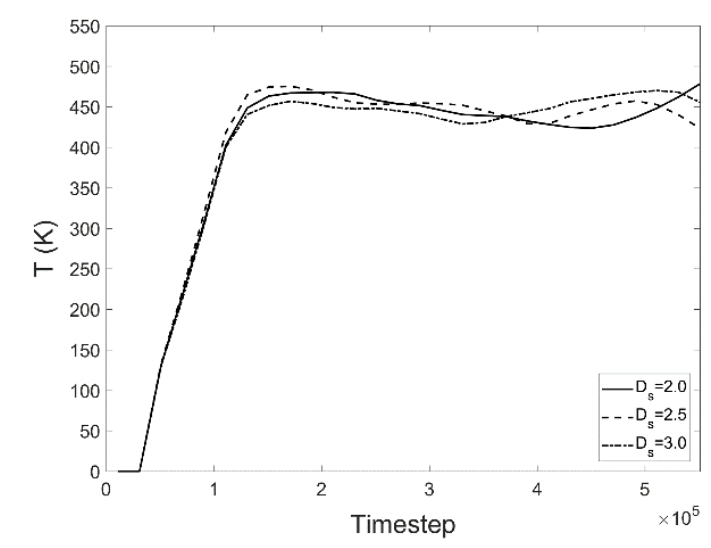

(b)

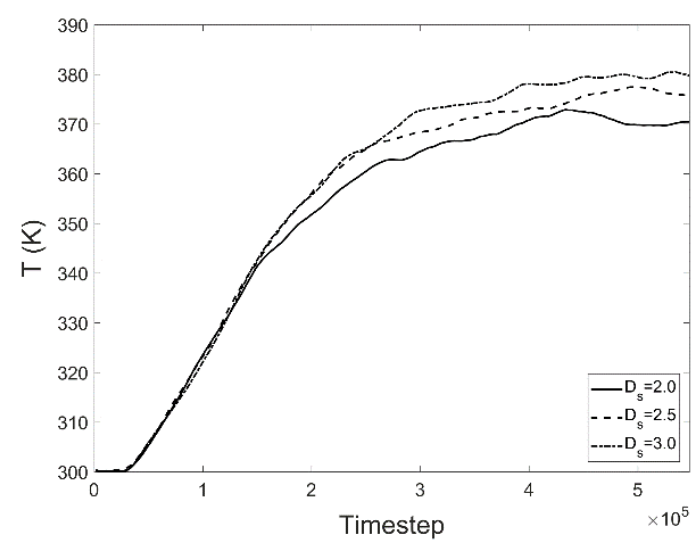

(d)

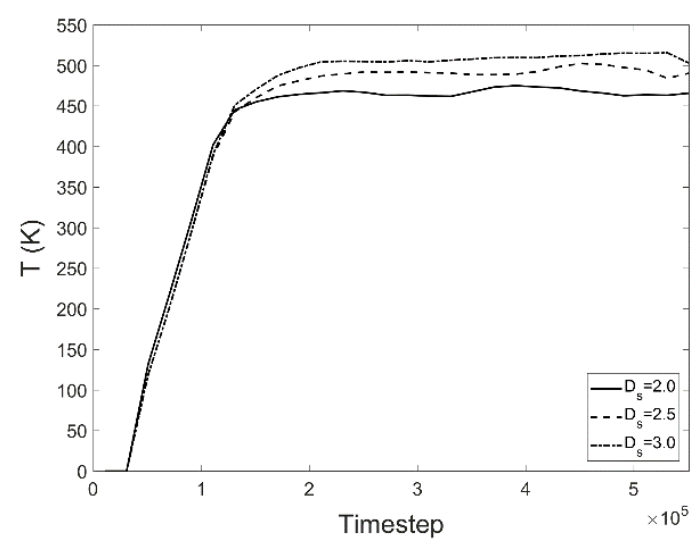

Figure 11: Temperature of the simulation box $(a \& b)$ and the vicinity of the abrasive $(c \& d)$ for the rough workpiece $(a \&$ c) and rough abrasive $(b \& d)$ cases vs time as a function of the fractal dimension of the abrasive surface. 
The statistically averaged temperature in the vicinity of the abrasive for rough workpiece and abrasive surfaces is illustrated in Figure 12. Although the average temperature is independent of the fractal dimension in the rough workpiece surface case, it increases approximately linearly with increasing fractal dimension of the abrasive surface.

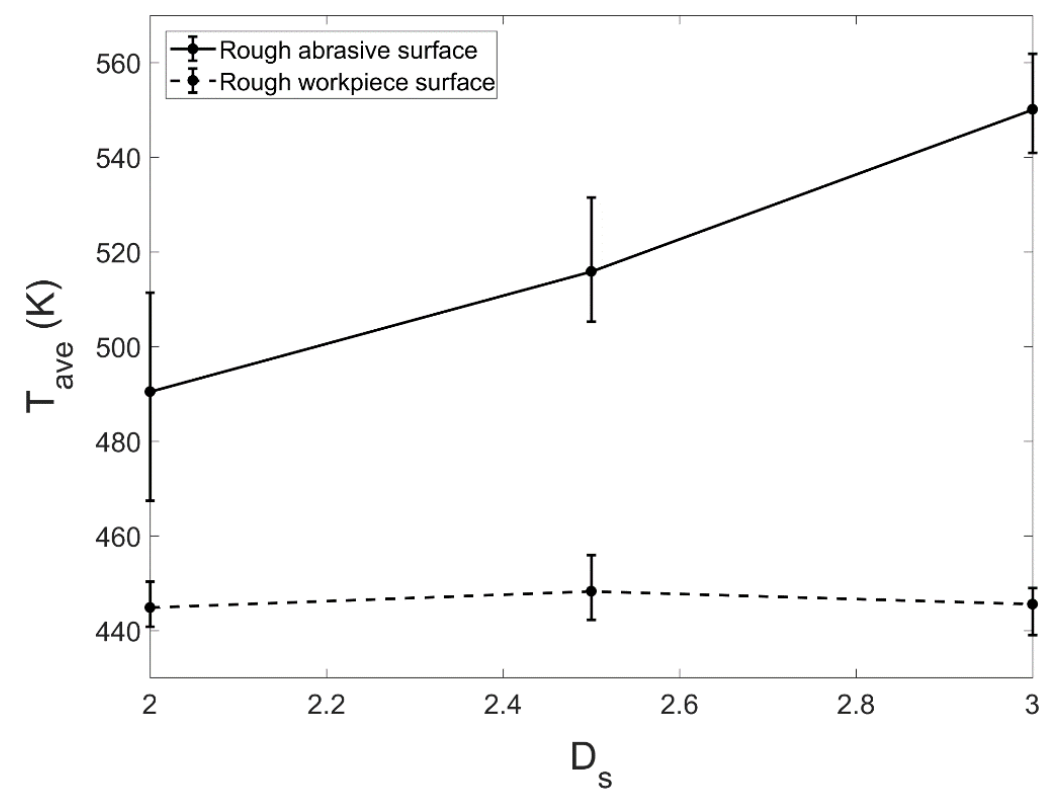

Figure 12: Average temperature for the rough workpiece and rough abrasive cases vs fractal dimension

Moreover, in Figure 12 we can observe that, when the interface between the workpiece and the abrasive is irregular, the subsurface temperature is always higher compared to the smooth interface case. This observation can be associated with the higher cutting forces observed when the abrasiveworkpiece interface is irregular (Figure 8) compared to the forces in the smooth interface case (Figure 6). As a matter of fact, higher cutting forces result in higher work and consequently higher heat dissipation across the simulation domain. This phenomenon is clearly illustrated in the 3-dimensional temperature profiles of Figure 13 which correspond to a cross section at $z=w / 2$, where $w$ is the width of the workpiece; the abrasive atoms have been hidden in favour of clarity. The temperature of each workpiece atom has been calculated based on the z-component of the atoms' velocities using Eq. 6, while its value has been averaged over a time window of 2,000 timesteps. The dark-coloured atoms at the bottom of each picture correspond to the boundary atoms, as illustrated in Figure 1, and hence, their temperature is equal to $0 \mathrm{~K}$. Both of the temperature profiles of Figure 13 correspond to an identical timestep $(t=420,000)$. It is apparent that, when the interface surface is rough (Figure 13b), the temperature of the workpiece atoms is higher in the vicinity of the abrasive compared to the smooth interface case (Figure 13a). This is more apparent in the region underneath the abrasive location. 
(a)

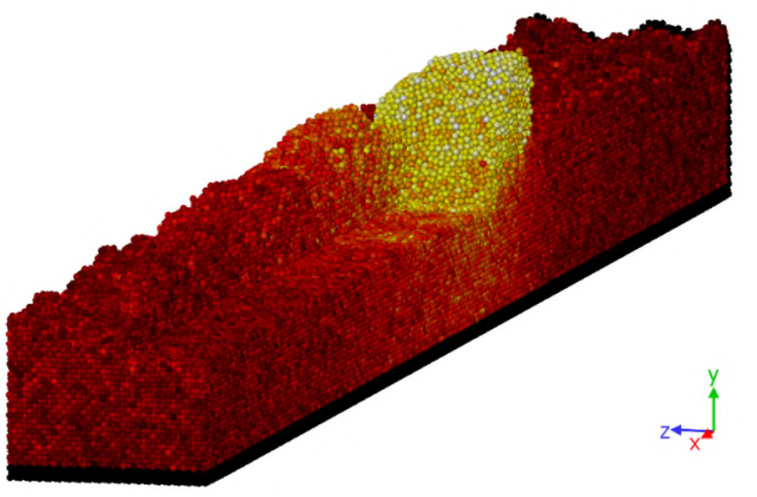

(b)

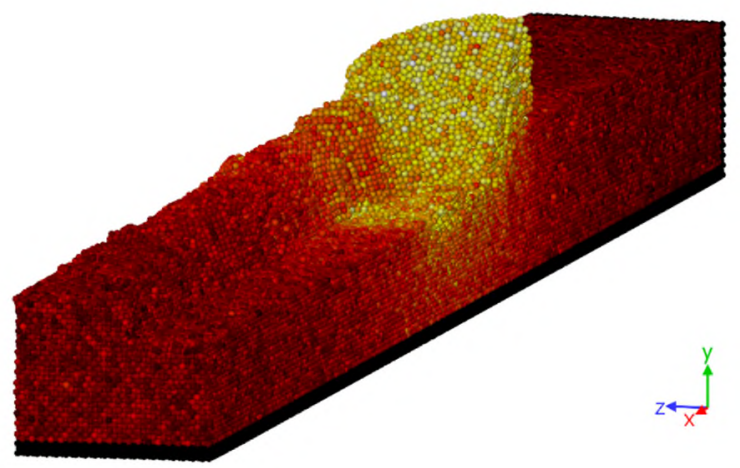

Figure 13: Temperature profile of the workpiece atoms for (a) rough workpiece and (b) rough abrasive simulation cases.

\subsection{Cutting depth effects on the grinding process characteristics}

In this section, the effect of cutting depth on the aforementioned process characteristics, namely grinding forces, friction coefficient and subsurface temperature, will be presented. Similarly to the previous sections, each data point corresponds to the average of 3 simulations with varying random phase of the rough profiles, while 3 different values of the depth of cut $\left(\mathrm{d}_{\mathrm{c}}\right)$ have been used based on the constraints imposed by the thickness of the workpiece: (a) $d_{c}=3 \mathrm{~nm}$, (b) $d_{c}=3.5 \mathrm{~nm}$, and (c) $d_{c}=4 \mathrm{~nm}$. When the workpiece surface is rough, the grinding forces are almost invariant to the fractal dimension but their magnitude increases for higher depth of cut (Figure 14). This is in agreement to previous studies [8].

(a)

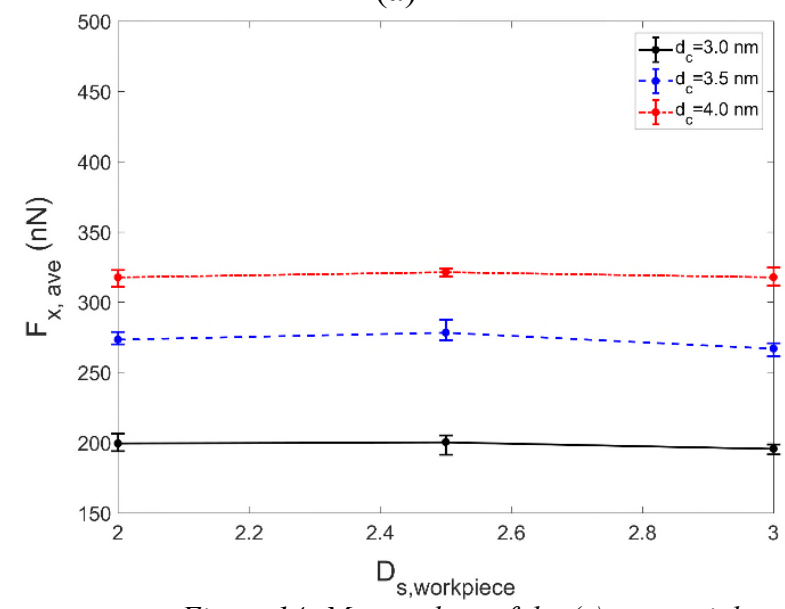

(b)

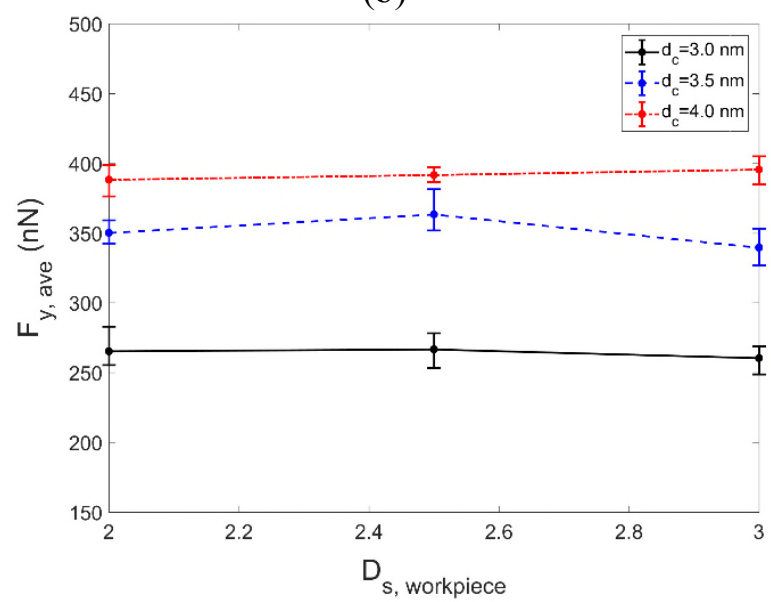

Figure 14: Mean values of the (a) tangential and (b) normal forces for the rough workpiece case 
As illustrated in Figure 15, when the abrasive surface is irregular both the tangential and normal forces present an increasing trend with the depth of cut and the values of the fractal dimension. At this point, it has to be highlighted that as the depth of cut increases, the increase of the normal force becomes in general steeper. This is attributed to the further compression of the two solids which in combination with the increasingly irregular interface leads to larger effective contact area. As pointed out in Paragraph 3.1.1, normal forces exhibit larger fluctuations compared to the tangential forces due to their higher magnitude. As a result, the corresponding error bars (Figure $14 \mathrm{~b} \&$ Figure $15 \mathrm{~b}$ ) are wider compared to the errorbars of the tangential forces (Figure 14a \& Figure 15a).

(a)

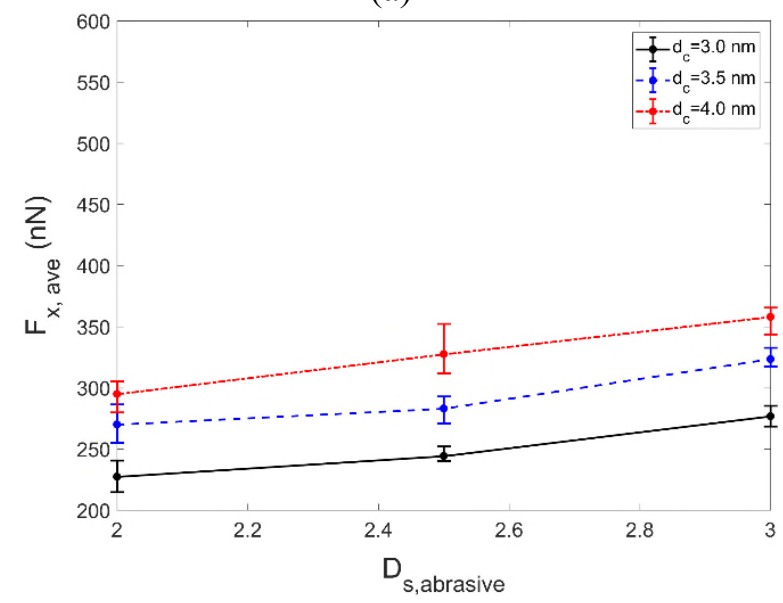

(b)

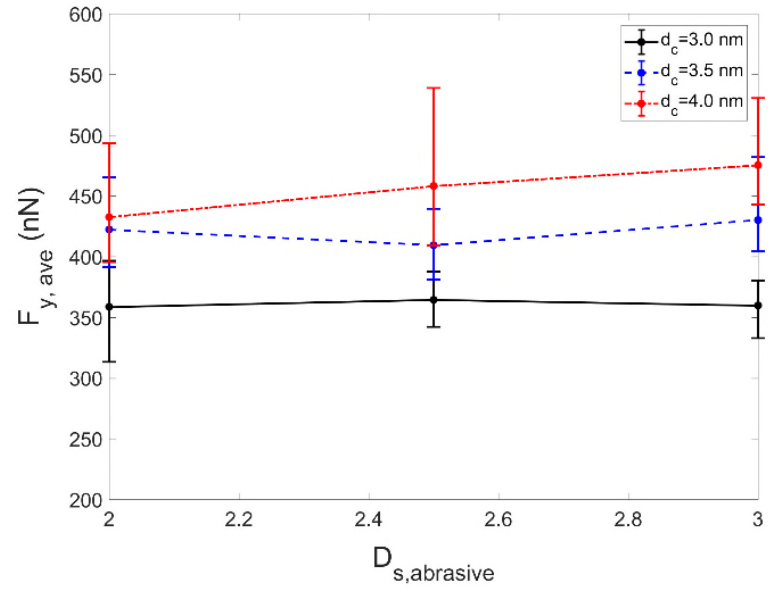

Figure 15: Mean values of the (a) tangential and (b) normal forces for the rough abrasive case

As expected, the friction coefficient is invariant to the value of the fractal dimension in the rough workpiece case (Figure 16a). Moreover, the friction coefficient increases with higher values of the depth of cut. This is in agreement with the results of Durgumahanti et al. [39] who performed singlegrit grinding experiments to develop a model for the estimation of the grinding forces. As illustrated in Figure $16 \mathrm{~b}$, when the abrasive surface is rough, the friction coefficient increases with the fractal dimension as well as the depth of cut. However, it is evident that for very irregular interfaces $\left(D_{s} \rightarrow 3\right)$ the effect of the fractal dimension becomes less significant. This is because higher values of the cutting depth increase the normal loading which is responsible for flattening the small scale irregularities associated with higher values of the fractal dimension [38]. Despite the wide errorbars in Figure 16, which are due to the fluctuation of the cutting forces as stated in the previous paragraph, the trends of the friction coefficient as a function of the fractal dimension and the cutting depth remain clear.

(a)

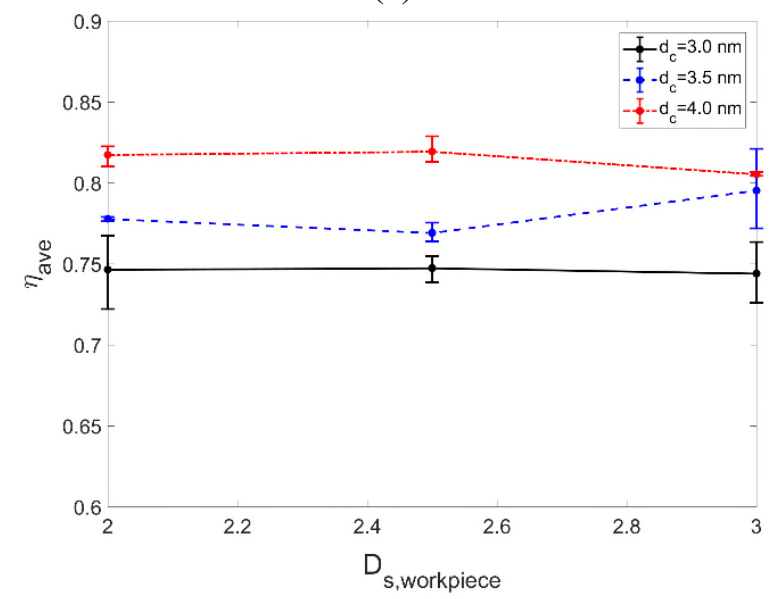

(b)

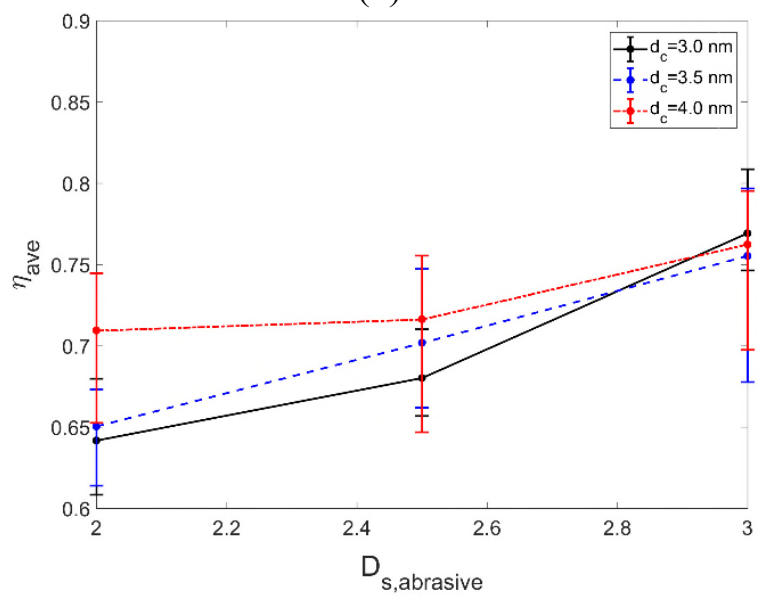

Figure 16: Mean friction coefficient vs fractal dimension for various cutting depth values: (a) rough workpiece surface, (b) rough abrasive surface 
Similarly to the friction coefficient, the workpiece temperature in the vicinity of the abrasive increases for higher values of the depth of cut for both the rough workpiece and rough abrasive cases, as illustrated in Figure 17. This is due to the larger contact area induced between the two solids. It can be observed that the increase of the subsurface temperature in the vicinity of the tool with the depth of cut is not linear but becomes weaker for higher values of the depth of cut. This is because as the depth of cut increases, the tool and consequently the moving region used for averaging approach the Langevin thermostat applied to the outmost layers of the workpiece; thus, the heat removal rate increases. This phenomenon becomes less distinct when the interface between the tool and the workpiece is rough due to the higher interlocking mechanism induced. The workpiece temperature is not affected by the workpiece fractal dimension but increases almost linearly with the fractal dimension of the abrasive surface. This is because in the first case (rough workpiece) the interface between the two solids is smooth, while in the second (rough abrasive) it is rough. As discussed in the previous subsection, rough interfaces contribute towards higher heat dissipation and consequently higher subsurface temperature.

(a)

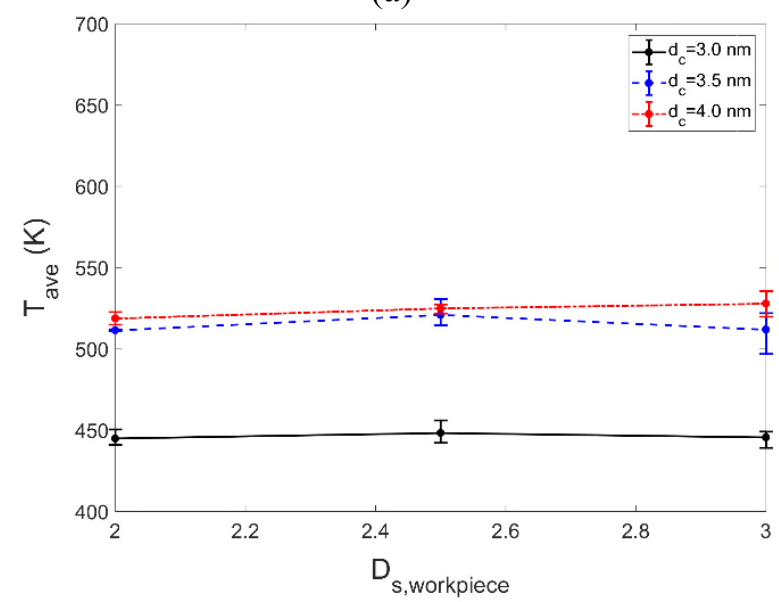

(b)

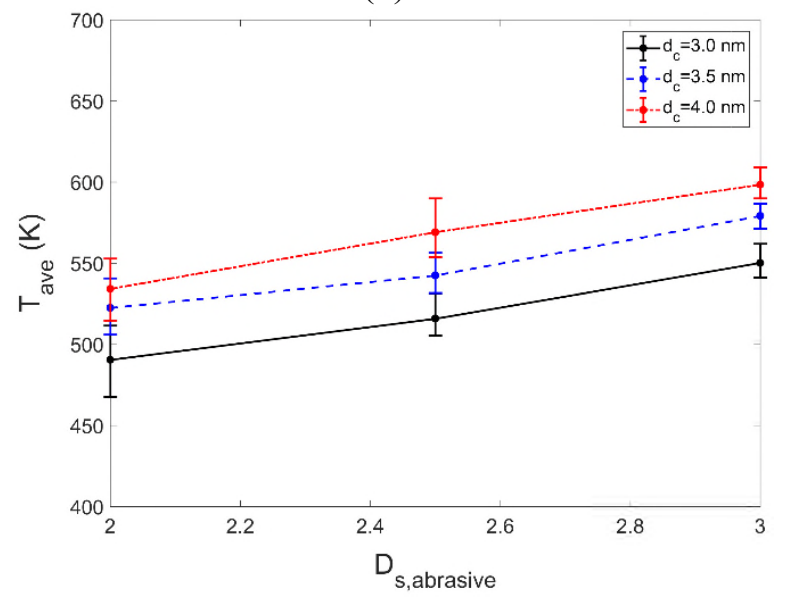

Figure 17: Mean friction coefficient vs fractal dimension for various cutting depth values: (a) rough workpiece surface, (b) rough abrasive surface

\section{Conclusions and Future Work}

In this investigation we have performed Molecular Dynamics simulations of grinding processes in order to investigate the surface roughness effects on the grinding mechanics. Surface roughness was modelled using the multivariate Weierstrass-Mandelbrot function and the surface irregularity was tuned by alternating the value of the fractal dimension $D_{s}$. The process parameters investigated were: (a) cutting forces, (b) friction coefficient and (c) workpiece temperature. We have studied the effects of the cutting depth in association with the workpiece top surface roughness and the abrasive lower surface roughness on the aforementioned process characteristics. Our results indicate that the grinding mechanics are sensitive to the cutting depth as well as the abrasive roughness topography, which also defines the interface between the two solids. On the contrary, the workpiece top surface does not affect the mechanics of the system. More analytically, the main conclusions drawn from this research are:

1. The topography of the upper workpiece surface does not significantly affect the grinding mechanics. The time-averaged values of the cutting forces, friction coefficient and workpiece 
temperature are independent of the workpiece top surface irregularities. This result is in agreement with previous experimental studies [36]. On the contrary, the topography of the abrasive lower surface significantly affects the grinding mechanics. This is attributed to the alternation of the workpiece-abrasive interface structure with the fractal dimension.

2. The cutting forces, friction coefficient and workpiece temperature increase with the depth of cut for both rough and smooth interface conditions. For higher values of the depth of cut the effect of the fractal dimension on the friction coefficient becomes less dominant due to the flattening of the small scale irregularities associated with higher values of the fractal dimension.

3. The rough interface between the workpiece and the abrasive leads to higher cutting forces, both normal and tangential, and consequently higher heat dissipation and subsurface temperature. Moreover, temperature profiles indicate that the hot spot areas are located at the chip and the interface regions.

4. Increased cutting forces associated with high values of the fractal dimension reduce the energy efficiency of the grinding process. Therefore, it is of high importance that the surface of the abrasive grains has the lowest possible fractal dimension $D_{s}$. This outcome points to the use of abrasive grains consisted of materials with diamond-like properties and surfaces characterised by low values of their fractal dimension or the implementation of nano-patterning techniques [25] for controlling the abrasive surface irregularity. 


\section{References}

[1] H. Van Brussel, J. Peirs, D. Reynaerts, A. Delchambre, G. Reinhart, N. Roth, M. Weck, E. Zussman, Assembly of Microsystems, CIRP Ann. 49 (2000) 451-472. doi:10.1016/S00078506(07)63450-7.

[2] E. Brinksmeier, J.C. Aurich, E. Govekar, C. Heinzel, H.-W. Hoffmeister, F. Klocke, J. Peters, R. Rentsch, D.J. Stephenson, E. Uhlmann, K. Weinert, M. Wittmann, Advances in Modeling and Simulation of Grinding Processes, CIRP Ann. 55 (2006) 667-696. doi:10.1016/J.CIRP.2006.10.003.

[3] W.L. Cooper, A.S. Lavine, Grinding Process Size Effect and Kinematics Numerical Analysis, J. Manuf. Sci. Eng. 122 (2000) 59. doi:10.1115/1.538888.

[4] J. Feng, P. Chen, J. Ni, Prediction of grinding force in microgrinding of ceramic materials by cohesive zone-based finite element method, Int. J. Adv. Manuf. Technol. 68 (2013) 10391053. doi:10.1007/s00170-013-4895-z.

[5] K. Salonitis, T. Chondros, G. Chryssolouris, Grinding wheel effect in the grind-hardening process, Int. J. Adv. Manuf. Technol. 38 (2008) 48-58. doi:10.1007/s00170-007-1078-9.

[6] A. Frangi, C. Cercignani, S. Mukherjee, N. Aluru, Advances in Multiphysics Simulation and Experimental Testing of Mems, Published by Imperial College Press and distributed by World Scientific Publishing co., 2008. doi:10.1142/p522.

[7] U. Landman, W.D. Luedtke, A. Nitzan, Dynamics of tip-substrate interactions in atomic force microscopy, Surf. Sci. Lett. 210 (1989) L177-L184. doi:10.1016/0167-2584(89)90800-1.

[8] R. Komanduri, N. Chandrasekaran, L.M. Raff, Effect of tool geometry in nanometric cutting: a molecular dynamics simulation approach, Wear. 219 (1998) 84-97. doi:10.1016/S00431648(98)00229-4.

[9] Q.X. Pei, C. Lu, F.Z. Fang, H. Wu, Nanometric cutting of copper: A molecular dynamics study, Comput. Mater. Sci. 37 (2006) 434-441. doi:10.1016/J.COMMATSCI.2005.10.006.

[10] P.M. Morse, Diatomic Molecules According to the Wave Mechanics. II. Vibrational Levels, Phys. Rev. 34 (1929) 57-64. doi:10.1103/PhysRev.34.57.

[11] M.S. Daw, M.I. Baskes, Embedded-atom method: Derivation and application to impurities, surfaces, and other defects in metals, Phys. Rev. B. 29 (1984) 6443-6453. doi:10.1103/PhysRevB.29.6443.

[12] Q.X. Pei, C. Lu, H.P. Lee, Large scale molecular dynamics study of nanometric machining of 
copper, Comput. Mater. Sci. 41 (2007) 177-185. doi:10.1016/J.COMMATSCI.2007.04.008.

[13] X. Guo, Q. Li, T. Liu, C. Zhai, R. Kang, Z. Jin, Molecular dynamics study on the thickness of damage layer in multiple grinding of monocrystalline silicon, Mater. Sci. Semicond. Process. 51 (2016) 15-19. doi:10.1016/J.MSSP.2016.04.013.

[14] J. Li, Q. Fang, L. Zhang, Y. Liu, The effect of rough surface on nanoscale high speed grinding by a molecular dynamics simulation, Comput. Mater. Sci. 98 (2015) 252-262. doi:10.1016/J.COMMATSCI.2014.10.069.

[15] H. Dai, G. Chen, S. Li, Q. Fang, B. Hu, Influence of laser nanostructured diamond tools on the cutting behavior of silicon by molecular dynamics simulation, RSC Adv. 7 (2017) 1559615612. doi:10.1039/C6RA27070K.

[16] B.B.-Y. Cao, M. Chen, Z.Z.-Y. Guo, Effect of surface roughness on gas flow in microchannels by molecular dynamics simulation, Int. J. Eng. Sci. 44 (2006) 927-937. http://www.sciencedirect.com/science/article/pii/S0020722506001042 (accessed January 20, 2016).

[17] N.V.N. Priezjev, S.S.M. Troian, Influence of periodic wall roughness on the slip behaviour at liquid/solid interfaces: molecular-scale simulations versus continuum predictions, J. Fluid Mech. 554 (2006) 25-46. http://journals.cambridge.org/abstract_S0022112006009086 (accessed November 8, 2016).

[18] W. Yan, K. Komvopoulos, Contact analysis of elastic-plastic fractal surfaces, J. Appl. Phys. 84 (1998) 3617-3624.

[19] S. Ganti, B.B.- Wear, undefined 1995, Generalized fractal analysis and its applications to engineering surfaces, Elsevier. (n.d.). https://www.sciencedirect.com/science/article/pii/0043164894065454 (accessed July 23, 2018).

[20] D. Avnir, D. Farin, P. Pfeifer, Molecular fractal surfaces, Nature. 308 (1984) 261-263. doi:10.1038/308261a0.

[21] Y. Chen, C. Zhang, M. Shi, G.G.P. Peterson, Slip boundary for fluid flow at rough solid surfaces, Appl. Phys. Lett. 100 (2012) 74102. http://scitation.aip.org/content/aip/journal/apl/100/7/10.1063/1.3685490 (accessed February 2, 2016).

[22] M. Papanikolaou, M. Frank, D. Drikakis, Nanoflow over a fractal surface, Phys. Fluids. 28 (2016) 082001. doi:10.1063/1.4958975. 
[23] M. Papanikolaou, M. Frank, D. Drikakis, Effects of surface roughness on shear viscosity, Phys. Rev. E. 95 (2017) 033108. doi:10.1103/PhysRevE.95.033108.

[24] C.K. Bora, E.E. Flater, M.D. Street, J.M. Redmond, M.J. Starr, R.W. Carpick, M.E. Plesha, Multiscale roughness and modeling of MEMS interfaces, Tribol. Lett. 19 (2005) 37-48. doi:10.1007/s11249-005-4263-8.

[25] A. Lehmann, C. Bradac, R.P. Mildren, Two-photon polarization-selective etching of emergent nano-structures on diamond surfaces, Nat. Commun. 5 (2014) 3341. doi:10.1038/ncomms4341.

[26] Z. Tong, Y. Liang, X. Jiang, X. Luo, An atomistic investigation on the mechanism of machining nanostructures when using single tip and multi-tip diamond tools, Appl. Surf. Sci. 290 (2014) 458-465. doi:10.1016/J.APSUSC.2013.11.113.

[27] H. Huang, Y.. Liu, Experimental investigations of machining characteristics and removal mechanisms of advanced ceramics in high speed deep grinding, Int. J. Mach. Tools Manuf. 43 (2003) 811-823. doi:10.1016/S0890-6955(03)00050-6.

[28] J. Tersoff, Empirical Interatomic Potential for Carbon, with Applications to Amorphous Carbon, Phys. Rev. Lett. 61 (1988) 2879-2882. doi:10.1103/PhysRevLett.61.2879.

[29] T. Inamura, N. Takezawa, N. Taniguchi, Atomic-Scale Cutting in a Computer Using Crystal Models of Copper and Diamond, CIRP Ann. 41 (1992) 121-124. doi:10.1016/S00078506(07)61166-4.

[30] L. Zhang, H. Tanaka, Towards a deeper understanding of wear and friction on the atomic scale - a molecular dynamics analysis, Wear. 211 (1997) 44-53. doi:10.1016/S00431648(97)00073-2.

[31] M. Ausloos, D.H. Berman, A multivariate Weierstrass-Mandelbrot function, Proc. R. Soc. London.A.Mathematical Phys. Sci. 400 (1985) 331-350. http://rspa.royalsocietypublishing.org/content/400/1819/331.short (accessed March 7, 2016).

[32] H.N. Li, T.B. Yu, Z.X. Wang, L. Da Zhu, W.S. Wang, Detailed modeling of cutting forces in grinding process considering variable stages of grain-workpiece micro interactions, Int. J. Mech. Sci. 126 (2017) 319-339. doi:10.1016/J.IJMECSCI.2016.11.016.

[33] B. Lin, S.Y. Yu, S.X. Wang, An experimental study on molecular dynamics simulation in nanometer grinding, J. Mater. Process. Technol. 138 (2003) 484-488. doi:10.1016/S09240136(03)00124-9.

[34] Q.X. Pei, C. Lu, H.P. Lee, Y.W. Zhang, Study of Materials Deformation in Nanometric 
Cutting by Large-scale Molecular Dynamics Simulations, Nanoscale Res. Lett. 4 (2009) $444-$ 451. doi:10.1007/s11671-009-9268-z.

[35] E.S. Mistakidis, O.K. Panagouli, Friction evolution as a result of roughness in fractal interfaces, Eng. Comput. 20 (2003) 40-57. doi:10.1108/02644400310458829.

[36] P.L. Menezes, Kishore, S. V. Kailas, Effect of Roughness Parameter and Grinding Angle on Coefficient of Friction When Sliding of Al-Mg Alloy Over EN8 Steel, J. Tribol. 128 (2006) 697. doi:10.1115/1.2345401.

[37] X. Cai, L. Yang, Z. Wang, M. Zhang, L. Shen, H. Hong, H. Lin, G. Yu, Influences of fractal dimension of membrane surface on interfacial interactions related to membrane fouling in a membrane bioreactor, J. Colloid Interface Sci. 500 (2017) 79-87. doi:10.1016/j.jcis.2017.03.107.

[38] O.K. Panagouli, K. Iordanidou, Dependence of friction coefficient on the resolution and fractal dimension of metallic fracture surfaces, Int. J. Solids Struct. 50 (2013) 3106-3118. doi:10.1016/J.IJSOLSTR.2013.05.020.

[39] U.S. Patnaik Durgumahanti, V. Singh, P. Venkateswara Rao, A New Model for Grinding Force Prediction and Analysis, Int. J. Mach. Tools Manuf. 50 (2010) 231-240. doi:10.1016/J.IJMACHTOOLS.2009.12.004. 
2018-10-20

Fractal roughness effects on nanoscale grinding

\author{
Papanikolaou, Michail
}

Elsevier

Papanikolaou M, Salonitis K. Fractal roughness effects on nanoscale grinding. Applied Surface

Science, Volumes 467-468, February 2019, pp. 309-319

https://doi.org/10.1016/j.apsusc.2018.10.144

Downloaded from Cranfield Library Services E-Repository 